\title{
ChemComm
}

\section{Gold nanorods embedded discotic nanoribbons $†$}

Cite this: Chem. Commun., 2013,

49, 978

Received 19th November 2012,

Accepted 11th December 2012

DOI: $10.1039 /$ c2cc38320a

\author{
B. S. Avinash, ${ }^{\text {ab }}$ V. Lakshminarayanan, ${ }^{a}$ Sandeep Kumar ${ }^{\star a b}$ and J. K. Vij ${ }^{\text {b }}$
}

www.rsc.org/chemcomm

It is shown that gold nanorods (GNRs) can be easily inserted into the supramolecular order of discotic liquid crystals (DLCs) along the director without disturbing their mesomorphism. GNRs embedded discotic nanoribbons, useful for constructing devices like thin film transistors, sensors, etc., are prepared by simple solution processing of GNR-DLC nanocomposites.

The self-assembling supramolecular columnar phases formed by discotic liquid crystals (DLCs) have been well recognized as onedimensional organic semiconductors useful in many devices like photovoltaic solar cells, light emitting diodes, thin film transistors, sensors, etc. ${ }^{1-18}$ On the other hand, gold nanorods (GNRs) have been extensively studied for their anisotropic properties and their applications not only in materials science but also in biosciences have been sought. ${ }^{19,20}$ Here it is shown that GNRs can be easily inserted into the supramolecular order of DLCs without disturbing their mesomorphism. GNRs embedded discotic nanoribbons are formed simply by solution processing. These DLC-GNR nanocomposites were characterized from UV-Vis spectroscopy, transmission electron microscopy (TEM), polarizing optical microscopy (POM), differential scanning calorimetry (DSC), X-ray diffraction analysis (XRD), scanning electron microscopy (SEM), scanning transmission electron microscopy (STEM) and conductivity studies. Results indicate insertion of GNRs into the discotic matrix along the director (the long axis parallel to the length of the ribbon). Such supramolecular architectures are useful for constructing devices like thin film transistors, sensors, etc.

Forming nanocomposites of nanoparticles with liquid crystals is an interesting field of study. Many groups have dispersed nanomaterials in the liquid crystalline system to align nanoparticles in an ordered manner while some others have functionalized nanoparticles with liquid crystalline molecules, ${ }^{21-24}$ and also used them

\footnotetext{
${ }^{a}$ Raman Research Institute, C.V. Raman Avenue, Sadashivanagar, Bangalore, 560080, India. E-mail: skumar@rri.res.in; Fax: +91 80 23610492; Tel: +9180 23610122

${ }^{b}$ Department of Electronic and Electrical Engineering, Trinity College, University of Dublin, Ireland. E-mail: jvij@tcd.ie

$\dagger$ Electronic supplementary information (ESI) available: Experimental section and Fig. S1 and S2. See DOI: 10.1039/c2cc38320a
}

as optical bulk metamaterials. ${ }^{25}$ We have previously doped the host discotics with carbonaceous, semiconducting and metallic nanoparticles and found interesting changes in their physical properties. ${ }^{26-30}$ A six order of magnitude conductivity enhancement was observed upon doping $1 \%$ of a discotic functionalized GNP in a triphenylene (TP) based DLC. ${ }^{29}$ Holt et al. also observed a similar enhancement in the conductivity of a TP DLC upon doping $1 \%$ of methylbenzene thiol coated GNPs. ${ }^{31}$ In this work we succeeded in incorporating the gold nanorods into hexapentyloxytriphenylene (HPT) DLC. Very recently, Liu et al. reported the dispersion of GNRs in a lyotropic LC. ${ }^{32}$ High conductivity obtained in these nanostructured systems will be of great importance in the field of organic electronics, as discotic molecules provide highly ordered structures through which charges created can diffuse out while the surface plasmon polaritons, generated on the nanoparticles due to incident light, can be used for plasmonic light concentration as well as for plasmonic light trapping. Previously, Wang et al. have formed nanowires of triphenylene by vapour deposition of triphenylene, ${ }^{33}$ similarly Ji et al. prepared nanowires of perylenediimide and naphthalenediimide. ${ }^{34}$ Here we show that such supramolecular triphenylene structure can be formed by a much simpler solution process. This provides an alternative to the vapour deposition technique wherein the sample has to be heated to higher temperatures, which would lead to decomposition of the organic molecules.

Gold nanorods were prepared following the literature method (see ESI $\dagger$ ) and characterized by UV-Vis spectroscopy and TEM (Fig. 1).

UV-Vis spectra of gold nanorods in aqueous medium have two peaks, a small peak at $515 \mathrm{~nm}$ and a peak at $733 \mathrm{~nm}$, which are transverse and longitudinal plasmon bands, respectively, typical of gold nanorods (Fig. 1b). ${ }^{35}$ The band at $515 \mathrm{~nm}$ has a shoulder band at $581 \mathrm{~nm}$, which is due to dog bone shape of gold nanorods which have slightly thicker edges compared to the middle portion. ${ }^{36}$ The TEM image of gold nanorods (Fig. 1a) shows that the nanorods prepared have an aspect ratio of 2.7 , and have dimensions of $40 \mathrm{~nm}$ in length and $15 \mathrm{~nm}$ in breadth.

Three composites viz., 1GNRTP, 2GNRTP, and 5GNRTP having $1 \%, 2 \%$ and 5\% GNR, respectively, in HPT were prepared by sonicating dichloromethane solution of two components 

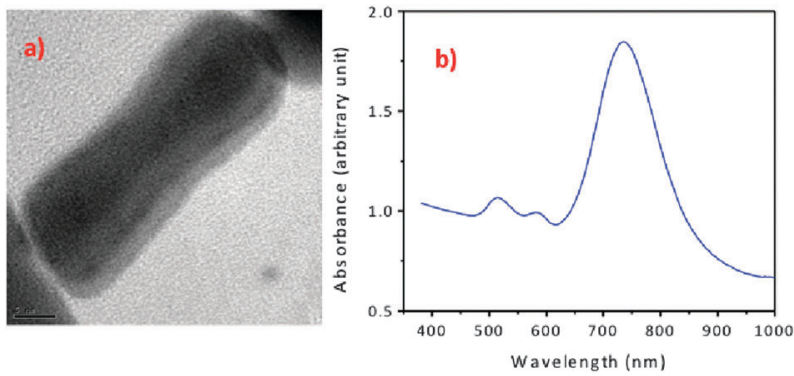

Fig. 1 (a) TEM image of gold nanorods, (b) UV-Vis spectra of gold nanorods in aqueous medium

followed by removal of solvent under vacuum. The UV-vis spectra of pure HPT and doped samples showed similar peaks (Fig. S1, ESI ${ }^{\dagger}$ ), indicating that the addition of gold nanorods does not change the absorption band of triphenylene systems. The plasmon resonance band of gold nanorods was not observed in the spectra of composites, as their concentration was very low.

The mesophase behaviour of the HPT and its composites was deduced by POM, DSC and XRD analysis. All the composites showed liquid crystalline behaviour, POM textures of the pure HPT as well as gold nanorod composites (Fig. 2) showed columnar hexagonal textures, and none of the composites showed any phase segregation indicating that gold nanorods are evenly dispersed in the mesophase.

Differential scanning calorimetry of composites was performed and compared with HPT to analyse the transition temperatures and the enthalpy associated with it (Fig. 3a). Table 1 lists the melting and clearing temperatures of HPT and its gold nanorod composites. The doped samples show a small increase in melting temperature compared to the pure HPT, while the clearing temperatures are not affected much. This observation shows that gold nanorods stabilize the peripheral alkyl chain melting without imparting any significant effect on core-core packing. Enhanced stability in melting transition could be due to van der Waals interaction between alkyl chains surrounding the discotics and dodecanethiol alkyl chains present on gold.

The X-ray diffraction patterns were recorded for HPT and all composites under the same conditions at $90{ }^{\circ} \mathrm{C}$. One-dimensional intensity $v s .2 \theta$ and diffraction patterns for pure HPT and

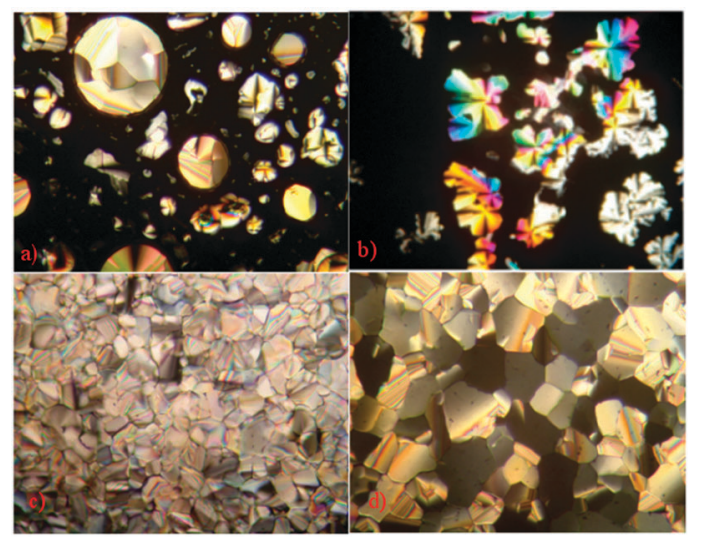

Fig. 2 POM textures of (a) HPT, (b) 1GNRTP, (c) 2GNRTP and (d) 5GNRTP. The photographs were taken under crossed polarisers at $90{ }^{\circ} \mathrm{C} ; 200 \times$ magnification.
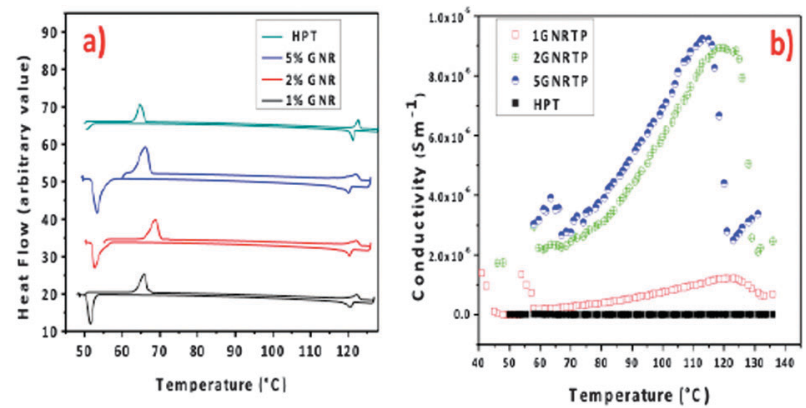

Fig. 3 (a) (DSC) curves of HPT (green), 1GNRTP (black), 2GNRTP (red) and 5GNRTP (blue); (b) conductivity of the samples HPT (black), 1GNRTP (red), 2GNRTP (green), 5GNRTP (blue).

Table 1 Transition temperatures and associated enthalpies of pure HPT and the gold nanorod composites

\begin{tabular}{lll}
\hline Sample & $\mathrm{Cr} \rightarrow$ Colh & Colh $\rightarrow \mathrm{I}$ \\
\hline HPT & $64.8^{\circ} \mathrm{C}\left(42.92 \mathrm{~J} \mathrm{~g}^{-1}\right)$ & $122.5^{\circ} \mathrm{C}\left(11.26 \mathrm{~J} \mathrm{~g}^{-1}\right)$ \\
1GNRTP & $65.8^{\circ} \mathrm{C}\left(42.46 \mathrm{~J} \mathrm{~g}^{-1}\right)$ & $122 .{ }^{\circ} \mathrm{C}\left(10.81 \mathrm{~J} \mathrm{~g}^{-1}\right)$ \\
2GNRTP & $68.8^{\circ} \mathrm{C}\left(41.04 \mathrm{~J} \mathrm{~g}^{-1}\right)$ & $122.1^{\circ} \mathrm{C}\left(8 \mathrm{~J} \mathrm{~g}^{-1}\right)$ \\
5GNRTP & $66.1{ }^{\circ} \mathrm{C}\left(42.12 \mathrm{~J} \mathrm{~g}^{-1}\right)$ & $122{ }^{\circ} \mathrm{C}\left(9.06 \mathrm{~J} \mathrm{~g}^{-1}\right)$
\end{tabular}

composites are shown in Fig. S2 (ESI $\dagger$ ); all the samples showed a similar plot with Bragg spacings of the first two reflections having a ratio of $1: 1 / \sqrt{ } 3$ typical of a columnar hexagonal phase. There is not much difference found between HPT and composites indicating that the insertion of GNRs does not destroy columnar arrangement of discs.

The undoped HPT sample shows a conductivity of $7.2 \times$ $10^{-9} \mathrm{Sm}^{-1}$ at $132{ }^{\circ} \mathrm{C}$, conductivity of the sample increased with an increase in temperature, and mesophase conductivity of HPT varies from $4.7 \times 10^{-10} \mathrm{Sm}^{-1}$ at $65^{\circ} \mathrm{C}$ to $4.5 \times 10^{-9} \mathrm{Sm}^{-1}$ at $122{ }^{\circ} \mathrm{C}$. The doped samples show much higher conductivity, 1GNRTP shows conductivity of $1.22 \times 10^{-6} \mathrm{Sm}^{-1}$ at $121{ }^{\circ} \mathrm{C}$ but above the isotropic phase conductivity decreases to $6 \times$ $10^{-7} \mathrm{Sm}^{-1}$. Samples 2GNRTP and 5GNRTP exhibit similar behaviour. As can be seen from Fig. 3b, increasing the concentration of GNRs increases the conductivity of the composite significantly. In all the cases the conductivity in the mesophase increases with an increase in temperature.

Discotic nanoribbons were formed when a solution of HPT or HPT-GNR composites in chloroform was added to methanol (see ESI + ). The resultant material was drop cast on a silicon substrate and a copper grid for SEM and STEM analysis respectively. The STEM images were recorded after about one month of formation of the composites (due to non-availability of the instrument), indicating that the nanostructure was stable for at least a month. Both HPT and gold nanorod composites showed long ribbon-like structures (Fig. 4) formed by coming together of many hexagonal columns. The non-polar alkyl chains surrounding the discs in a polar solvent drive the columnar structure formed by individual discs to come together and form supramolecular aggregates. This leads columnar structures to form a supramolecular ribbon-like structure. These results are in accordance with literature reports. ${ }^{37-39}$ Xiao et al., ${ }^{37}$ Che et $a .^{38}$ and Koshkakaryan et $a l^{39}$ have clearly demonstrated the formation of nanoribbons composed of discotic molecules with the 

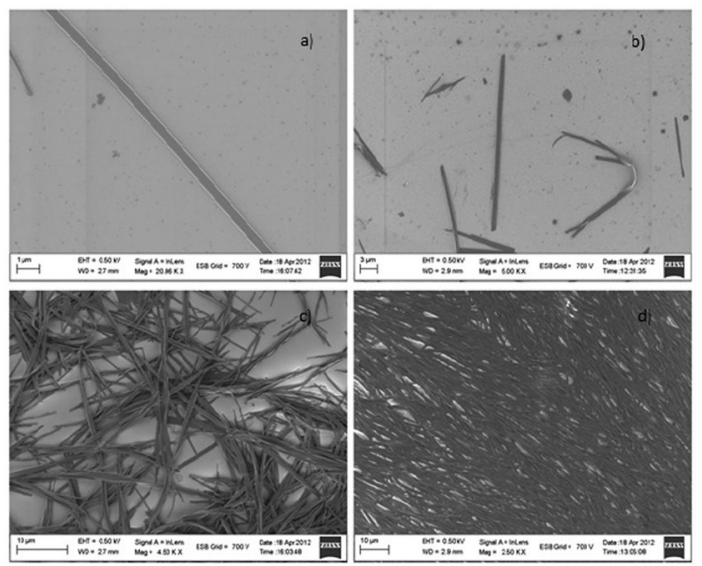

Fig. 4 SEM images of (a) single nanoribbon of HPT, (b) nanoribbons formed by 1 GNRTP, (c) randomly oriented ribbons of HPT and (d) bundles of ribbons formed by 1 GNRTP.

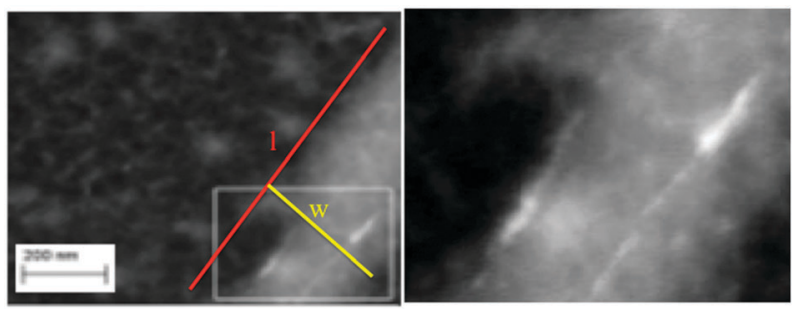

Fig. 5 Dark field STEM images of gold nanorods embedded in HPT nanoribbons; (a) bright spots in the fibres are due to gold nanorods embedded in the ribbon with their longitudinal axes parallel to the length of the ribbon (I represents the length of the fibre while $w$ stands for the width), (b) the magnified portion of (a) showing gold nanorods of length $\sim 40 \mathrm{~nm}$ in ribbons.

$\pi$ planes stacking in the direction of the long axis of the ribbon. While Che et al. ${ }^{38}$ reported high photoconductivity in the nanoribbons, Xiao et $a .^{37}$ fabricated a transistor device using the nanofibers. In our GNRTP nanoribbons, the GNRs can align along the length, width or thickness of the nanoribbons. The dark field STEM image of 5GNRTP nanoribbons (Fig. 5) clearly shows that gold nanorods are aligned with their long axes parallel to the length of the ribbon. GNRs of length $\sim 40 \mathrm{~nm}$, same as that observed in TEM, can be clearly seen along the length of the fibers, no bright spots corresponding to the length or width of GNRs are observed along the width of the fibers.

In conclusion, we have shown that hexpentyloxytriphenylene primarily self-assembles into hexagonal structures, which upon proper solution processing can be made to grow into ribbonlike secondary structures. During the growth, these supramolecular structures can trap the gold nanorods present in the solution. Thus forming supramolecular nanocomposites, which show enhanced conductivity owing to insertion of nanorods in ribbon-like structures.

SFI Grant No. 11/W.1/I1957 is gratefully acknowledged.

\section{Notes and references}

1 S. Kumar, Chemistry of Discotic Liquid Crystals: From Monomers to Polymers, CRC Press, Boca Raton, FL, 2011.

2 R. J. Bushby and K. Kawata, Liq. Cryst., 2011, 38, 1415-1426.

3 H. K. Bisoyi and S. Kumar, Liq. Cryst., 2011, 38, 1427-1449.

4 B. R. Kaafarani, Chem. Mater., 2011, 23, 378-396.

5 W. Pisula, X. Feng and K. Mullen, Chem. Mater., 2011, 23, 554-567.

6 H. K. Bisoyi and S. Kumar, Chem. Soc. Rev., 2010, 39, 264-285.

7 S. Kumar, Liq. Cryst., 2009, 36, 607-638.

8 J. W. Goodby, I. M. Saez, S. J. Cowling, V. Gortz, M. Draper, A. W. Hall, S. Sia, G. Cosquer, S.-E. Lee and E. P. Raynes, Angew. Chem., Int. Ed., 2008, 47, 2754-2787.

9 J. Wu, W. Pisula and K. Müllen, Chem. Rev., 2007, 107, 718-747.

10 S. Laschat, A. Baro, N. Steinke, F. Giesselmann, C. Hägele, G. Scalia, R. Judele, E. Kapatsina, S. Sauer, A. Schreivogel and M. Tosoni, Angew. Chem., Int. Ed., 2007, 46, 4832-4887.

11 S. Sergeyev, W. Pisula and Y. Geerts, Chem. Soc. Rev., 2007, 36, 1902-1929.

12 C. Tschierske, Chem. Soc. Rev., 2007, 36, 1930-1970.

13 S. Kumar, Chem. Soc. Rev., 2006, 35, 83-109.

14 T. Kato, N. Mizoshita and K. Kishimoto, Angew. Chem., Int. Ed., 2006, 45, 38-68.

15 B. Donnio, D. Guillon, R. Deschenaux and D. W. Bruce, in Comprehensive Coordination Chemistry II, ed. J. A. McCleverty and T. J. Meyer, Elsevier, Oxford, 2003, vol. 7, pp. 357-627.

16 K. Ohta, K. Hatsusaka, M. Sugibayashi, M. Ariyoshi, K. Ban, F. Maeda, R. Naito, K. Nishizawa, A. M. van de Craats and J. M. Warman, Mol. Cryst. Liq. Cryst., 2003, 397, 25-45.

17 N. Boden, R. J. Bushby, J. Clements and B. Movaghar, J. Mater. Chem., 1999, 9, 2081-2086.

18 A. N. Cammidge and R. J. Bushby, in Handbook of Liquid Crystals, ed. D. Demus, J. Goodby, G. W. Gray, H. W. Spiess and V. Vill, Wiley-VCH, Weinheim, 1998, ch. VII, vol. 2B, pp. 693-748.

19 X. Huang, S. Neretina and M. A. El-Sayed, Adv. Mater., 2009, 21, $4880-4910$.

20 C. J. Murphy, L. B. Thompson, D. J. Chernak, J. A. Yang, S. T. Sivapalan, S. P. Boulos, J. Huang, A. M. Alkilany and P. N. Sisco, Curr. Opin. Colloid Interface Sci., 2011, 16, 128-134.

21 G. L. Nealon, R. Greget, C. Dominguez, Z. T. Nagy, D. Guillon, J.-L. Gallani and B. Donnio, Beilstein J. Org. Chem., 2012, 8, 349-370.

22 O. Stamatoiu, J. Mirzaei, F. Xiang and T. Hegmann, Top. Curr. Chem., 2012, 318, 331-393.

23 H. K. Bisoyi and S. Kumar, Chem. Soc. Rev., 2011, 40, 306-319.

24 J. Milette, S. J. Cowling, V. Toader, C. Lavigne, I. M. Saez, R. B. Lennox, J. W. Goodby and L. Reven, Soft Matter, 2012, 8, 173-179.

25 R. Pratibha, K. Park, I. Smalyukh and W. Park, Opt. Express, 2009, 17, 19459-19469.

26 S. Kumar and V. Lakshminarayanan, Chem. Commun., 2004, 1600-1601.

27 S. Kumar and H. K. Bisoyi, Angew. Chem., Int. Ed., 2007, 46, 1501-1503.

28 S. Kumar, S. K. Pal and V. Lakshminarayanan, Mol. Cryst. Liq. Cryst., 2005, 434, 251-258.

29 S. Kumar, S. K. Pal, P. S. Kumar and V. Lakshminarayanan, Soft Matter, 2007, 3, 896-900.

30 S. Kumar and L. Sagar, Chem. Commun., 2011, 47, 12182-12186.

31 L. A. Holt, R. J. Bushby, S. D. Evans, A. Burgess and G. Seeley, J. Appl. Phys., 2008, 103, 063712.

32 Q. Liu, B. Senyuk, J. Tang, T. Lee, J. Qian, S. He and I. Smalyukh, Phys. Rev. Lett., 2012, 109, 088301.

33 H. Wang, X. Xu, A. Kojtari and H. Ji, J. Phys. Chem. C, 2011, 115, 20091-20096.

34 H.-F. Ji, R. Majithia, X. Yang, X. Xu and K. More, J. Am. Chem. Soc., 2008, 130, 10056-10057.

35 B. Nikoobakht and M. A. El-Sayed, Chem. Mater., 2003, 15, 1957-1962.

36 L. Gou and C. J. Murphy, Chem. Mater., 2005, 17, 3668-3672.

37 S. Xiao, J. Tang, T. Beetz, X. Guo, N. Tremblay, T. Siegrist, Y. Zhu, M. Steigerwald and C. Nuckolls, J. Am. Chem. Soc., 2006, 128, 10700.

38 Y. Che, X. Yang, G. Liu, C. Yu, H. Ji, J. Zuo, J. Zhao and L. Zang, J. Am. Chem. Soc., 2010, 132, 5743.

39 G. Koshkakaryan, P. Jiang, V. Altoe, D. Cao, L. M. Klivansky, Y. Zhang, S. Chung, A. Katan, F. Martin, M. Salmeron, B. Ma, S. Aloni and Y. Liu, Chem. Commun., 2010, 46, 8579. 